\title{
2016 David P. Robbins Prize
}

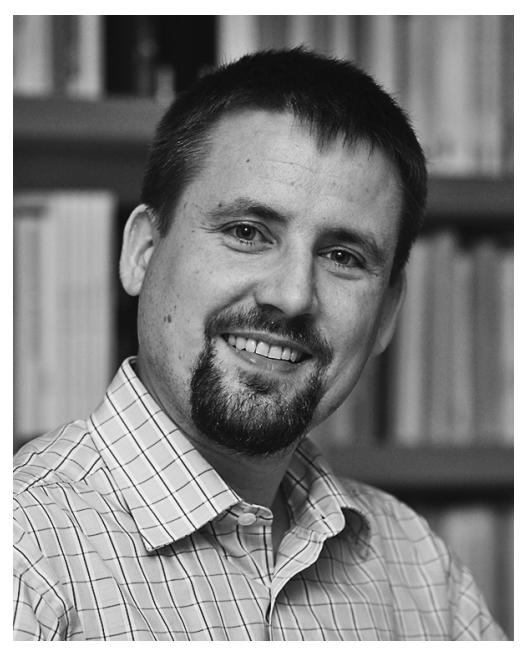

Christoph Koutschan

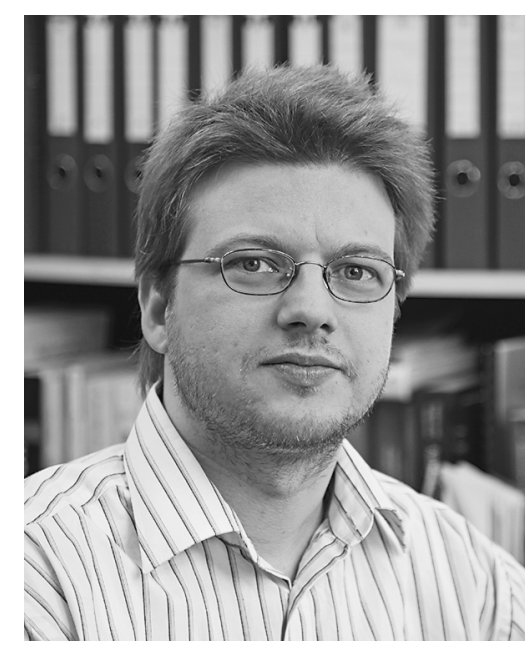

Manuel Kauers

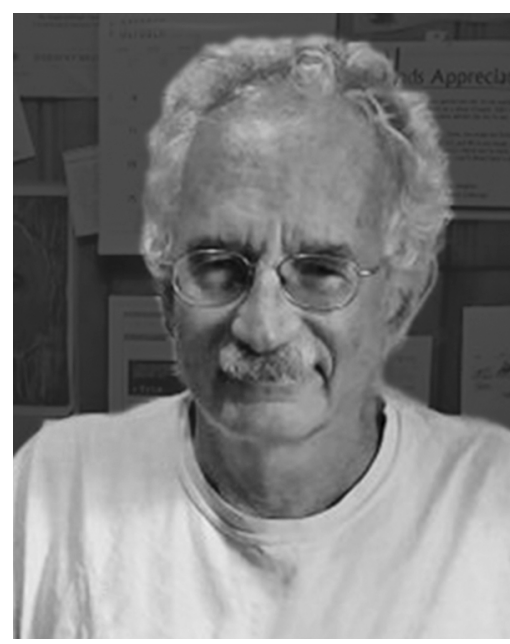

Doron Zeilberger
Christoph Koutschan, Manuel Kauers, and DORON ZEILBERGER were awarded the 2016 David P. Robbins Prize at the 122nd Annual Meeting of the AMS in Seattle, Washington, in January 2016 for their paper, "Proof of George Andrews's and David Robbins's q-TSPP conjecture," Proceedings of the National Academy of Sciences of the USA, 108 (2011), 2196-2199.

\section{Citation}

A plane partition $\pi$ may be thought of as a finite set of triples of natural numbers with the property that if $(i, j, k)$ is in $\pi$, then so is every $\left(i^{\prime}, j^{\prime}, k^{\prime}\right)$ with $i^{\prime} \leq i, j^{\prime} \leq j$, and $k^{\prime} \leq k$. The symmetric group $S_{3}$ acts on plane partitions by permuting the coordinate axes. A plane partition $\pi$ may also be "complemented" by constructing the set of points not in $\pi$ that are in the smallest box enclosing $\pi$, and then reflecting them through the center of the box. The set of plane partitions that is invariant under some subgroup of these transformations is known as a "symmetry class of plane partitions."

In the early 1980s, the combined experimental observations of several people, including David Robbins himself, suggested that all the

For permission to reprint this article, please contact: reprint-permission@ams.org.

DOI: http://dx.doi.org/10.1090/noti1356 symmetry classes of plane partitions were enumerated by a family of remarkably simple product formulas. In some cases, this was also found to be true of the $q$-analogs of these formulas-generating functions where $q$ tracks some parameter of interest. Simple combinatorial formulas usually have simple combinatorial explanations, but surprisingly that is not the case here. Many of the formulas are exceedingly difficult to prove, and to this day there is no unified proof that explains why they are all so simple and so similar. When simple combinatorial formulas fail to have simple combinatorial proofs, it usually means that something deep is going on beneath the surface. Indeed, the study of symmetry classes of plane partitions has revealed unexpected connections between several areas, including statistical mechanics, the representation theory of quantum groups, alternating sign matrices, and lozenge tilings.

By 1995 all the formulas had been proved but one: the $q$-TSPP (totally symmetric plane partitions, where $q$ tracks the number of orbits of triples) formula, independently conjectured by George Andrews and David Robbins. The paper by Koutschan, Kauers, and Zeilberger, along with supporting computer files on Koutschan's website, finally established the correctness of this 
last conjectured formula. It is a tour de force of experimental mathematics.

The authors' starting point was a 1989 paper of Okada that reduced the problem to a conjecture on the values of the determinants of a special sequence of matrices. The form of the matrices enables one to verify the conjecture by Zeilberger's "holonomic ansatz," in which one hypothesizes a system of auxiliary functions that would certify the correctness of the determinant evaluation. If these auxiliary functions exist, then one can search for them computationally. Once found, the functions must be shown to satisfy certain recurrences in order to complete the proof. The authors performed the search, found the candidate auxiliary functions, and empirically verified that they seemed to satisfy the necessary recurrences. Although techniques existed that might in principle find proofs for the recurrences, the necessary computations seemed far beyond the reach of current computers, and the authors initially announced a semirigorous but not fully rigorous proof. However, the authors then developed "creative telescoping" techniques that dramatically simplified the necessary computations, resulting in a fully rigorous proof of the $q$-TSPP formula.

The David P. Robbins Prize is awarded to a novel research paper in algebra, combinatorics, or discrete mathematics with a significant experimental component. The $q$-TSPP paper is a shining example. The conjecture itself was born from experimental observations, and the proof involved the development of new experimental mathematical techniques that are sure to solve many problems.

\section{Biographical Sketch of Koutschan}

Christoph Koutschan, born in 1978, received his master's degree in 2005 from Friedrich Alexander University and his PhD in 2009 from the Johannes Kepler University under the direction of Peter Paule. He was a postdoctoral fellow at the Research Institute for Symbolic Computation (RISC, Linz), at Tulane University, and at the Institut National de Recherche en Informatique et en Automatique (INRIA). He is currently with the Johann Radon Institute for Computational and Applied Mathematics (RICAM) of the Austrian Academy of Sciences. His research interests are devoted to computer algebra methods related to the holonomic systems approach, particularly symbolic summation and integration algorithms, and their application in other areas of mathematics.

\section{Response from Christof Koutschan}

I'm deeply honored to receive the David P. Robbins Prize for the proof of the $q$-enumeration formula for totally symmetric plane partitions. This beautiful and mysterious formula was conjectured independently by David Robbins and George Andrews in the early 1980s. It was none other than Doron Zeilberger who proposed to my colleague Manuel Kauers and me to tackle this problem, which he constantly called "the holy grail of enumerative combinatorics." Back then I was a PhD student and quite excited to work on such an old and important conjecture. At the very beginning I didn't know at all what a totally symmetric plane partition was or what $q$-enumeration meant. But thanks to Soichi Okada, who had reformulated the conjecture in terms of a family of determinants, that wasn't even necessary; the only thing one had to do was to evaluate these determinants. And Doron had already devised a recipe - the holonomic ansatz-how one could do that, but the necessary computer algebra calculations seemed to be far beyond what was possible with the current hardware and software. As a warmup example we applied Doron's recipe to the $q=1$ case, first proved by John Stembridge in 1995 using a different approach, but already this considerably easier task appeared to be hopeless. At this point, the visionary suggestion of my $\mathrm{PhD}$ advisor Peter Paule to investigate and implement the holonomic systems approach turned out to be a lucky chance: by the end of my PhD I had found a way to solve the $q=1$ case, and, shortly after, a new method for computing creative telescoping relations was born, which was efficient enough to prove the determinant evaluations that implied the $q$-TSPP formula. Since that time this method, which was originally motivated from David Robbins's conjecture, has been successfully applied to various other problems ranging from enumerative combinatorics and statistical physics to numerical analysis and knot theory.

\section{Biographical Sketch of Kauers}

Manuel Kauers was born in Germany and studied computer science at the University of Karlsruhe from 1998 to 2002 before he went to Linz, Austria, to do a PhD in symbolic computation under the supervision of Peter Paule. He earned his doctoral degree in 2005 and an habilitation in mathematics in 2008. In 2009, he was awarded the START prize of the Austrian federal minister for science and education, the most prestigious award for young scientists in Austria, endowed with a research grant of 1.2 million euros. In June 2015, Kauers was appointed full professor at the Johannes Kepler University in Linz, where he is now the director of the Institute for Algebra. He is best known for his contributions to computer algebra and its applications to combinatorics and experimental mathematics.

\section{Response from Manuel Kauers}

Back in 2006, Doron Zeilberger visited Linz and sketched a way to prove the $q$-TSPP conjecture computationally. The computations he suggested were quite expensive, and there was no reason (other than his intuition) that the "certificate" he aimed to compute would even exist. We were reluctant to invest time and energy on such a risky project, but Zeilberger kept pushing us until we did. Now in 2016, I am glad that he insisted, and 
I am happy to receive the David P. Robbins Prize together with Koutschan and Zeilberger for a hard piece of work that we have done together. I also appreciate very much that the committee has decided to recognize a paper with a proof that was found by computer algebra, thereby helping to raise the awareness of the power of today's computational methods, not just for industrial applications but also for pure mathematics.

\section{Biographical Sketch of Zeilberger}

Doron Zeilberger was born on July 2, 1950, in Haifa, Israel, to Ruth (Alexander) and Yehudah Zeilberger. He received his $\mathrm{PhD}$ in 1976 from the Weizmann Institute of Science (as a student of Harry Dym (a student of Henry McKean (a student of William Feller (a student of Richard Courant (a student of David Hilbert))))). He is currently the Board of Governors Professor at Rutgers University. In 1979 he married Jane D. Legrange (PhD, Physics, Illinois, 1980, currently at LGSInnovation). Their children are Celia (b. 1983), Tamar (b. 1986), and Hadas (b. 1990). In 1998 he received, with Herbert S. Wilf (1931-2012), the Steele Prize for Seminal Contributions to Research and, in 2004, the Euler Medal from the Institute of Combinatorics and Its Applications.

\section{Response from Doron Zeilberger}

David Peter Robbins's beautiful gravestone, in the Princeton Cemetery (here is a picture: www. math.rutgers.edu/ zei 1berg/graves/DaveRobbinsGrave.htm7) says that he was a "Solver of Problems." True, of course, but he was just as great a "Poser of Problems," and many of his problems kept me, and many of my colleagues, busy for many years, and some of them are still wide open. Dave was a pioneer of what is now called "experimental mathematics", and it is particularly gratifying that the work that I and my collaborators, Christoph Koutschan and Manuel Kauers, did to deserve the prize that bears his name uses his methodology of experimental mathematics, not just to conjecture interesting results, but also to prove them.

\section{About the Prize}

The David P. Robbins Prize was established in 2005 in memory of David P. Robbins by members of his family. Robbins, who died in 2003, received his $\mathrm{PhD}$ in 1970 from the Massachusetts Institute of Technology. He was a long-time member of the Institute for Defense Analysis Center for Communications Research and a prolific mathematician whose work (much of it classified) was in discrete mathematics.

The prize is given for a paper published during the preceding six calendar years that (1) reports on novel research in algebra, combinatorics, or discrete mathematics, (2) has a significant experimental component, (3) is on a topic broadly accessible, and (4) provides a simple statement of the problem and clear exposition of the work. The US $\$ 5,000$ prize is awarded every three years.

The David P. Robbins Prize is awarded by the AMS Council acting on the recommendation of a selection committee. The members of the 2016 David P. Robbins Prize Committee were the following individuals.

- Sara C. Billey

- Timothy Y. Chow

- Curtis Greene

- Victor Reiner

- Daniel A. Spielman (Chair)

The complete list of recipients of the David P. Robbins Prize follows.

2007 Samuel P. Ferguson, Thomas C. Hales

2010 Ileana Streinu

2013 Alexander Razborov

2016 Christoph Koutschan, Manuel Kauers, Doron Zeilberger 